\title{
A Linear Programming Approach to Routing Control in Networks of Constrained Nonlinear Positive Systems with Concave Flow Rates
}

\author{
Heather Arneson ${ }^{\mathrm{a}}$, Nicolas Dousse ${ }^{\mathrm{b}}$, Cédric Langbort ${ }^{\mathrm{c}}$ \\ a Aviation Systems Division, NASA Ames Research Center \\ PO Box 1/MS 210-6, Moffett Field, CA 94035-0001 \\ b Laboratory of Intelligent Systems, Ecole Polytechnique Fédérale de Lausanne (EPFL), \\ EPFL-STI-IMT-LIS, Station 11, 1015 Lausanne, Switzerland \\ c Department of Aerospace Engineering, University of Illinois at Urbana-Champaign, \\ 306 Talbot Lab/MC-236, 104 S. Wright St. Urbana, IL, 61801
}

\begin{abstract}
We consider control design for positive compartmental systems in which each compartment's outflow rate is described by a concave function of the amount of material in the compartment. We address the problem of determining the routing of material between compartments to satisfy time-varying state constraints while ensuring that material reaches its intended destination over a finite time horizon. We give sufficient conditions for the existence of a time-varying state-dependent routing strategy which ensures that the closed-loop system satisfies basic network properties of positivity, conservation and interconnection while ensuring that capacity constraints are satisfied, when possible, or adjusted if a solution cannot be found. These conditions are formulated as a linear programming problem. Instances of this linear programming problem can be solved iteratively to generate a solution to the finite horizon routing problem. Results are given for the application of this control design method to an example problem.
\end{abstract}

Key words: linear programming; control of networks; positive systems; controller constraints and structure.

\section{Introduction}

Positive compartmental systems are popular models for describing interconnections of reservoirs whose dynamics are governed by conservation laws and natural positivity and capacity constraints. Examples include automobile or air traffic flows, job-balancing in computer clusters [5], or irrigation networks [2], to name just a few.

A specific class of such compartmental systems, known as Eulerian models, has been used extensively in the air

* This work was funded in part by a NASA Illinois Space Grant Consortium seed grant and NSF grant \#0826469 to C. Langbort, and University of Illinois College of Engineering SURGE Fellowship, and NASA Aeronautics Scholarship Program Fellowship to H. Arneson.

Email addresses: heather.arneson@nasa.gov (Heather Arneson), nicolas.dousse@epfl.ch (Nicolas Dousse), langbort@illinois.edu (Cédric Langbort). traffic management (ATM) literature $[1,4,7,8,10,11$, 14, 15]. In an Eulerian model, the aggregate dynamics of groups of aircraft are modeled instead of the dynamics of each individual aircraft. As a result, the order of an Eulerian model depends only on the number of compartments or sections used to describe the network of interest, but not on the total number of vehicles, which greatly reduces complexity in many cases. For more details on Eulerian models and their comparison to Lagrangian models, the reader is referred to [15].

Previous work in this area $[1,10,11,14,15]$ has focused primarily on the use of linear models to describe the outflow of each compartment, or section, of the network. Such models describe the outflow rate of a section as depending linearly on the amount of material in that section.

Here, we focus on concave outflow rate functions. Examples motivating the use of this type of outflow model 
come from road traffic and air traffic management research. Commonly used models for road traffic rely on a concave flow rate function to describe traffic flow as a function of density. The flow rate increases to a certain point, beyond which, traffic slows, eventually to a stop, as density increases. For example, the cell transmission model for highway traffic introduced in [3] uses a triangular fundamental flow diagram relating density to flow rate. More recently, a triangular outflow rate model was also used in the development of an analytical solution to the traffic flow model in [9].

Unlike road traffic, air traffic cannot slow to a stop as aircraft must maintain a minimum speed. The authors of [8] point out that, although the outflow of a section of airspace will increase as the density of traffic increases, there is an upper bound on the outflow rate. At low density, flights are allowed to traverse a given section of airspace at their nominal speed and thus more aircraft in a given section results in a greater outflow rate from that section. At low traffic density, it is reasonable to assume a linear relationship between the number of aircraft in the section and the outflow rate of that section. However, aircraft must maintain a minimum separation for safety considerations, and thus, as traffic density increases, separation requirements cause the outflow rate to saturate. A nonlinear, saturating outflow model is proposed in [8] and is shown to more accurately capture this saturating effect in dense traffic problems. In that work, the authors generate an increasing, concave, saturating outflow rate curve empirically through a discrete-event simulation which includes separation requirements.

Air traffic flow management is currently performed by human air traffic controllers. Traffic management coordinators (TMCs) react to flow constraints in the $\mathrm{Na}$ tional Airspace System (NAS) by implementing air traffic management initiatives in order to safely route air traffic through the NAS. Decisions are made based on TMCs' previous experience with similar conditions affecting a specific region of airspace. Each TMC is likely to react differently given identical conditions. Unfortunately, decisions made in this way are often overly conservative and do not include guarantees on performance. Air traffic is expected to increase steadily over the next two decades [12]. Air traffic controllers will increasingly rely on automation of this process in order to keep up with the growing demand.

Our previous work $[1,4]$ has focused on variations of the problem of routing design for positive compartmental systems to satisfy time-varying capacity constraints motivated by problems in air traffic management. Airspace sector capacity specifies the maximum number of aircraft that a trained human air traffic controller can safely route through the sector. Sector capacity depends on controller workload associated with traffic flow patterns in the sector. Additionally, the number of aircraft that can safely be routed through a sector of airspace at a given time can depend on the weather conditions in the sector at that time. Work has been done to estimate and predict sector capacities [13] in the presence of severe weather conditions.

In [1], we presented a solution to this problem for single destination networks with linear section outflow rates. The extension of this control design technique to the multiple destination problem is straightforward. Given linear outflow rates, a separate, decoupled network can be created for each destination. Routing solutions can be found individually for each sub-network using the technique presented in [1].

We focused on single destination networks with nonlinear section outflow rates in [4]. In contrast to the linear outflow problem of [1], extending this technique to the multiple destination problem is not straightforward because, with nonlinear outflow rates, sub-networks for each destination exhibit nonlinear coupling. This nonlinear coupling makes the derivation of routing solutions more challenging.

Here, we extend our former work and present a solution to the problem for a multiple destination network with nonlinear outflow rates. Rather than formulating constraints, and subsequently a linear programming (LP) problem, to solve this problem directly, we make use of the solution for the multiple destination network with linear outflow rates. Additional constraints are imposed on the routing solution for the latter so that the resulting closed-loop system with linear outflow rates behaves like the system with nonlinear section outflow rates. These constraints are nonlinear in control design variables and thus cannot be incorporated into the LP problem. To address this issue, we treat these constraint values as fixed and iteratively solve instances of the LP problem, adjusting the fixed values at each iteration.

While our motivation is the application of this control design technique to problems in air traffic management for which saturating outflow rates arise naturally, the control technique is applicable to any concave outflow rate function. We thus derive the control design technique for the more general concave outflow rate function with the saturating outflow rate function being a special case.

The multiple destination network with nonlinear outflow rates, control design objectives which ensure that basic network properties hold, and the formal problem statement are presented in Section 2. The derivation of the control design technique is presented in several stages in Section 3. First, the extension of the control design technique of [1] for the multiple destination network with linear section outflow rates, formulated as an LP problem, is described in Section 3.1. Control design for the multiple destination network with nonlinear outflow rates is 
then developed in Section 3.2. Constraints on the control input which force the multiple destination linear outflow rate system to behave like the multiple destination nonlinear outflow rate system are developed and incorporated as additional constraints in the LP problem of Section 3.1. A method to recover a routing strategy for the nonlinear outflow rate system from a solution to this modified LP problem is given. Finally, an algorithm is presented to iteratively solve instances of this modified LP problem. In Section 4, an application example is given to demonstrate the proposed solution method.

Notation: We are often concerned with vectors, scalars and elements of matrices which are indexed over some range. Therefore, for every positive integer $N$, we define $[N]=\{1,2, \ldots, N\}$. The cone of entry-wise nonnegative vectors of dimension $N$ is denoted by $\mathbb{R}_{+}^{N}$. We write $\boldsymbol{x} \geq 0$ to mean that $\boldsymbol{x} \in \mathbb{R}_{+}^{N}$, and $\boldsymbol{x}>0$ if it is in its interior.

\section{Problem Description}

\subsection{Model}

We consider positive conservative systems, which can be used to describe the flow of material through a network of interconnected sections. Sections may represent, for example, reservoirs in an irrigation network, portions of the road in a traffic model, or volumes of air space in an air traffic flow management problem. Material flows between these sections making its way from one of several sources to a particular sink.

We begin by describing the dynamics of an $N$-section network with $R$ distinct destinations or sinks. In addition to satisfying any performance objective, the routing solution must also ensure that all material reaches its intended destination. We aggregate material within each section of the network based on final destination. That is, assuming that there are $R$ distinct destinations, we create $R$ coupled sub-networks. Each sub-network describes the flow of traffic through the $N$-section network for a particular destination $r \in[R]$.

Some of the sections, referred to as "final sections," are sinks through which material exits the network. The set of final sections for each destination $r$ will be denoted by $\mathcal{S}_{\mathcal{F}}^{r}$. It is assumed that the network consists of at least one sink for each destination, that is $\mathcal{S}_{\mathcal{F}}^{r} \neq \emptyset$ for all $r \in[R]$.

The subset of sections into which material in a section $i \in[N]$ bound for destination $r$ can flow is denoted by $\mathcal{O}_{i}^{r}$. Since it may not be possible for material to reach a specific destination from any section in the network, we specify the set $\mathcal{N}^{r} \subseteq[N]$ for all $r \in[R]$ as the set of sections $i \in[N]$ such that there exists at least one path from section $i$ to a section in $\mathcal{S}_{\mathcal{F}}^{r}$. That is, there exist a sequence of sections $i_{1}, i_{2}, \ldots, i_{p}$ such that $i_{1}=i$, $i_{l+1} \in \mathcal{O}_{i_{l}}^{r}$ for $l=1,2, \ldots, p-1, i_{l} \in \mathcal{N}^{r} \backslash \mathcal{S}_{\mathcal{F}}^{r}$ for all $l<p$, and $i_{p} \in \mathcal{S}_{\mathcal{F}}^{r}$. We assume that the connectivity of all networks of interest satisfy $\mathcal{O}_{i}^{r} \subseteq \mathcal{N}^{r}$ for all $i \in[N]$, which ensures that material bound for destination $r$ is routed into sections which are connected to destination $r$. Networks satisfying this property are said to be outflow connected.

Flow control is applied by modifying the outflow rate of sections of the network. Physically, this flow control corresponds to holding or slowing down the material moving through the section. Mathematically, this can be described as taking a portion of the outflow of the section and re-routing it back into the section from which it exited. Thus, the mathematical descriptions of the networks we consider have self-loops and $i \in \mathcal{O}_{i}^{r}$ for all $i \in \mathcal{N}^{r}$ and $r \in[R]$.

The state of section $i$ of sub-network $r$ at time $t$, which represents the amount of material in section $i$ bound for destination $r$ at time $t$, is denoted by $x_{i}^{r}(t)$. The dynamics of this state is given by

$$
\begin{aligned}
\dot{x}_{i}^{r}(t)= & -\frac{x_{i}^{r}(t)}{\mathrm{x}_{i}(t)} \mu_{i}\left(\mathrm{x}_{i}(t)\right) \\
& +\sum_{j: i \in \mathcal{O}_{j}^{r}} \beta_{j i}^{r}(t) \frac{x_{j}^{r}(t)}{\mathrm{x}_{j}(t)} \mu_{j}\left(\mathrm{x}_{j}(t)\right) \\
& +\sum_{s=1}^{S} d_{s i}^{r}(t)
\end{aligned}
$$

where the meanings of the various symbols are summarized in Table 1 .

Outflow functions $\mu_{i}: \mathbb{R}_{+} \rightarrow \mathbb{R}_{+}$satisfy the following assumptions

$$
\begin{aligned}
& \mu_{i}(0)=0, \\
& \mu_{i} \text { is differentiable at } 0, \\
& \mu_{i} \text { is concave, }
\end{aligned}
$$

for all $i \in[N]$. Expression (1) is developed under the assumption (as is done, e.g., in [10]) that material bound for each destination is distributed uniformly throughout each section, so that the portion of the outflow of section $i$ bound for destination $r$ is given by

$$
\frac{x_{i}^{r}(t)}{\mathrm{x}_{i}(t)} \mu_{i}\left(\mathrm{x}_{i}(t)\right) .
$$

Note that, although physically relevant for some applications, the assumption that $\mu_{i}$ is saturating, that is

$$
\lim _{a_{i} \rightarrow+\infty} \mu_{i}\left(a_{i}\right)<\infty
$$


Table 1

Variable definitions

$$
\begin{aligned}
& \text { Full state of sub-network } r \text { : } \\
& \boldsymbol{x}^{r}(t)=\left(x_{1}^{r}(t), x_{2}^{r}(t), \ldots, x_{N}^{r}(t)\right)^{T} \in \mathbb{R}_{+}^{N} \\
& \mathrm{x}_{i}(t)=\sum_{r=1}^{R} x_{i}^{r}(t) \\
& \mathbf{x}(t)=\sum_{r=1}^{R} \boldsymbol{x}^{r}(t) \\
& =\left(\mathrm{x}_{1}(t), \mathrm{x}_{2}(t), \ldots, \mathrm{x}_{N}(t)\right)^{T} \in \mathbb{R}_{+}^{N} .
\end{aligned}
$$

Fraction of flow from $i$ to $j$ bound for $r$ :

$$
\beta_{i j}^{r}(t)
$$

Set of routing parameters for sub-network $r$ :

$$
\boldsymbol{\beta}^{r}(t)=\left\{\beta_{i j}^{r}(t)\right\}_{i, j}
$$

Set of routing parameters for full network:

$$
\boldsymbol{\beta}(t)=\left\{\beta_{i j}^{r}(t)\right\}_{i, j, r}
$$

Flow rate from source $s$ to section $i$ bound for $r$ :

$$
d_{s i}^{r}(t)
$$

Inflow rate of source $s$ bound for $r$ :

$$
\boldsymbol{d}_{s}^{r}(t)=\left(d_{s 1}^{r}(t), d_{s 2}^{r}(t), \ldots, d_{s N}^{r}(t)\right)^{T} \in \mathbb{R}_{+}^{N}
$$

Flow rate out of section $i$

$$
\mu_{i}\left(\mathrm{x}_{i}(t)\right)
$$

Flow rates of full network:

$$
\boldsymbol{\mu}(\mathbf{x}(t))=\left(\mu_{1}\left(\mathrm{x}_{1}(t)\right), \mu_{2}\left(\mathrm{x}_{2}(t)\right), \ldots, \mu_{N}\left(\mathrm{x}_{N}(t)\right)\right)^{T}
$$

for all $i \in[N]$, is not required for the application of the method and is thus not included in the above assumptions.

Given the assumption that $\mu_{i}$ is differentiable at 0 , and that $\mu_{i}(0)=0$,

$$
\lim _{\mathrm{x}_{i}(t) \rightarrow 0} \frac{\mu_{i}\left(\mathrm{x}_{i}(t)\right)}{\mathrm{x}_{i}(t)}=\frac{d \mu_{i}}{d \mathrm{x}_{i}}(0)
$$

and thus the outflow rate given in (3) remains finite even as $\mathrm{x}_{i}(t)$ approaches 0 .

An example network consisting of 21 sections, three distinct sinks and three sources is shown in Figure 1(a). Arrows indicate allowable flow between sources, sections and sinks. Recirculation is allowed in all sections. Final sections are 19, 20 and 21. The outflow of section 19 flows into sink 1 , the outflow of section 20 flows into sink 2 and the outflow of section 21 flows into sink 3 and thus $\mathcal{S}_{\mathcal{F}}^{1}=\{19\}, \mathcal{S}_{\mathcal{F}}^{2}=\{20\}$, and $\mathcal{S}_{\mathcal{F}}^{3}=\{21\}$. Given that there are three distinct sinks in this problem, the full network is divided into three sub-networks. Sub-networks

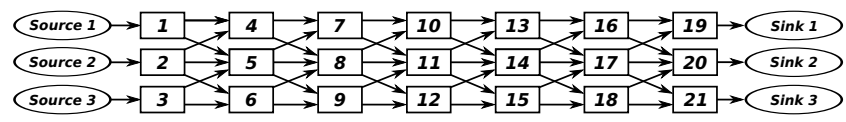

(a) Full network.

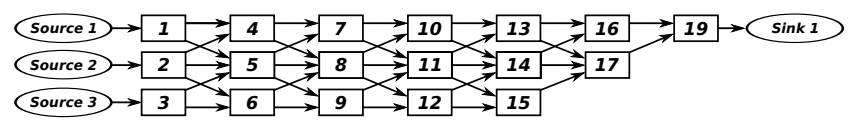

(b) Sub-network for destination 1.

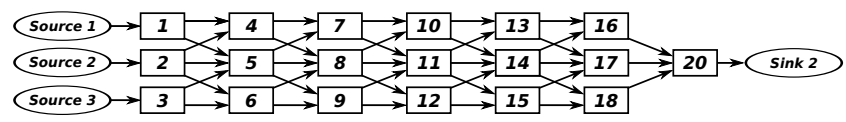

(c) Sub-network for destination 2.

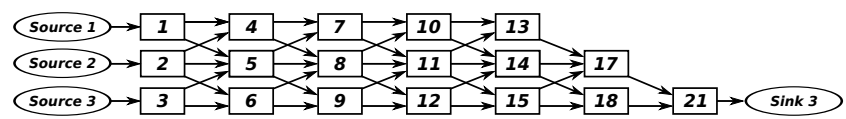

(d) Sub-network for destination 3.

Fig. 1. Figures 1(a), 1(b), 1(c), and 1(d) depict the connectivity of the full network and sub-networks associated with destination 1, 2 and 3, respectively. The number in each box indicates the index of that section. Arrows indicate allowable flow between sections of the network or sub-network Recirculation is allowed for all sections.

for destinations 1, 2 and 3 are given in Figures 1(b), 1(c) and $1(\mathrm{~d})$, respectively. Notice that each sub-network has fewer than 21 sections. This is due to the fact that specific destinations are unreachable from certain sections in the full network. For instance, in the sub-network associated with destination 1, shown in Figure 1(b), it is not possible to travel from sections 18,20 and 21 to sink 1 given the section interconnection of the full network. These sections belong to the set $[N] \backslash \mathcal{N}^{1}$ and are omitted from the destination 1 sub-network.

The dynamics of material in sub-network $r$ can be written compactly as

$$
\begin{aligned}
\dot{\boldsymbol{x}}^{r}(t)= & \left(\boldsymbol{\beta}^{r}(t)^{T}-\boldsymbol{I}\right) \operatorname{diag}\left(\boldsymbol{\mu}(\mathbf{x}(t)) \operatorname{diag}(\mathbf{x}(t))^{-1} \boldsymbol{x}^{r}(t)\right. \\
& +\sum_{s=1}^{S} \boldsymbol{d}_{s}^{r}(t)
\end{aligned}
$$

for all $r \in[R]$ with initial state values

$$
\boldsymbol{x}^{r}(0)=\boldsymbol{x}_{0}^{r},
$$

where $\boldsymbol{x}_{0}^{r}=\left(x_{1,0}^{r}, x_{2,0}^{r}, \ldots, x_{N, 0}^{r}\right)$. The initial state and inflow rates must satisfy $\left(\boldsymbol{x}_{0}^{r}\right)_{i} \geq 0$ for all $i \in \mathcal{N}^{r},\left(\boldsymbol{x}_{0}^{r}\right)_{i}=$ 0 for all $i \in[N] \backslash \mathcal{N}^{r}$, and $d_{s i}^{r}(t)=0$ for all $t \geq 0$ for all $i \in[N] \backslash \mathcal{N}^{r}$.

We denote the solution to (4) with initial state $\boldsymbol{x}^{r}(0)=$ $\boldsymbol{x}_{0}^{r}$ for all $r \in[R]$ and inflow rates $\boldsymbol{d}_{s}^{r}(t)$ for all $s \in[S]$ and $r \in[R]$, under the particular choice of routing strategy $\boldsymbol{\beta}(t)$, as $\mathbf{x}^{\beta}(t)$. 


\subsection{Basic Control Design Objectives}

The model introduced in Section 2.1 describes the flow of material through a network of sections. In order to be physically meaningful, this model must satisfy the following constraints:

Positivity: Given initial state $\boldsymbol{x}_{0}^{r} \geq 0$ for all $r \in[R]$ and inflow rate $\boldsymbol{d}_{s}^{r}(t) \geq 0$ for all $s \in[S], r \in[R]$ and $t \geq 0$, the resulting state vector satisfies

$$
\boldsymbol{x}^{r}(t) \geq 0
$$

for all $r \in[R]$ and $t \geq 0$.

Conservation: Constraints $\mathcal{C}\left(\boldsymbol{\beta}^{r}(t)\right)$ are defined for all $r \in[R], t \geq 0$ by

$$
\begin{aligned}
\beta_{i j}^{r}(t) & \geq 0, \forall i, j, \in \mathcal{N}^{r} \\
\beta_{i j}^{r}(t) & =0, \forall j \in \mathcal{N}^{r} \backslash \mathcal{O}_{i}^{r}, \forall i \in \mathcal{N}^{r} \\
\sum_{j \in \mathcal{O}_{i}^{r}} \beta_{i j}^{r}(t) & =1, \forall i \in \mathcal{N}^{r} \backslash \mathcal{S}_{\mathcal{F}}^{r} \\
\sum_{j \in \mathcal{O}_{i}^{r}} \beta_{i j}^{r}(t) & \leq 1, \forall i \in \mathcal{S}_{\mathcal{F}}^{r} .
\end{aligned}
$$

Note that constraints $\mathcal{C}\left(\boldsymbol{\beta}^{r}(t)\right)$ do not include terms $\beta_{i j}^{r}(t)$ for $i \notin \mathcal{N}^{r}$ or $j \notin \mathcal{N}^{r}$. It is not necessary to enforce constraints on these terms as they do not appear in the dynamic model (4). When required, individual constraints included in the set $\mathcal{C}\left(\boldsymbol{\beta}^{r}(t)\right)$ will be referred to using subscripts. For example, constraint (5) will be referred to by $\mathcal{C}_{1}\left(\boldsymbol{\beta}^{r}(t)\right)$.

Positivity ensures that the state of each section of every sub-network, which represents a physical quantity, remains non-negative at all times. Physically, the Conservation requirement ensures that material leaving a given non-final section enters another section in the network and is thus conserved. When designing the control input, care must be taken to ensure that the system satisfies these two conditions.

Conservation is satisfied when $\mathcal{C}\left(\boldsymbol{\beta}^{r}(t)\right)$ hold for all $r \in[R]$. Given non-negative routing parameters, that is, routing parameters satisfying $\mathcal{C}_{1}\left(\boldsymbol{\beta}^{r}(t)\right)$, constraints $\mathcal{C}_{3}\left(\boldsymbol{\beta}^{r}(t)\right)$, ensure that material exiting a non-final section is routed to a subsequent section in the network. Note that constraints $\mathcal{C}_{4}\left(\boldsymbol{\beta}^{r}(t)\right)$, defined for final sections only, allow material to exit the network.

Again, given non-negative routing parameters, the Positivity constraint is satisfied when the section outflow, $\mu_{i}$ for all $i$, satisfies certain conditions, detailed in the following claim.
Claim 1 Let initial condition $\boldsymbol{x}_{0}^{r} \geq 0$ for all $r \in[R]$, inflow rates $d_{s}^{r}(t) \geq 0$ for all $s \in[S], r \in[R], t \geq 0$, and routing parameters $\beta_{i j}^{r}(t) \geq 0$ be given for all $i, j \in$ $\mathcal{N}^{r}, r \in[R], t \geq 0$. Then, if each outflow rate function $\mu_{i}$ satisfies assumptions (2a) and (2b) and

$$
\mu_{i}(a) \geq 0 \text { for all } a \geq 0,
$$

for all $i \in[N]$, the solution of system (4) satisfies $\boldsymbol{x}^{r}(t) \geq$ 0 for all $r \in[R]$ and $t \geq 0$ thus Positivity holds, and consequently $\mathbf{x}(t) \geq 0$ for all $t \geq 0$.

PROOF. Given that $\boldsymbol{x}_{0}^{r} \geq 0$ for all $r \in[R]$, in order to show that $\boldsymbol{x}^{r}(t)$ remains positive for all $r \in[R]$ and $t \geq 0$, it is sufficient to show that for any $i \in \mathcal{N}^{p}$ and $p \in \bar{\epsilon}[R]$

$$
\left.\begin{array}{l}
x_{i}^{p}(t)=0 \\
\boldsymbol{x}^{r}(t) \geq 0, \forall r \in[R]
\end{array}\right\} \Rightarrow \dot{x}_{i}^{p}(t) \geq 0
$$

With $\dot{x}_{i}^{p}(t)$ given by (4) and assuming that $x_{j}^{r}(t) \geq 0$ for all $j \in \mathcal{N}^{r}$ and $r \in[R]$, and, in turn, $\mathrm{x}_{l}(t) \geq 0$ for all $l \in[N]$, it is clear that the only potentially negative component of $\dot{x}_{i}^{p}(t)$ is $-\frac{x_{i}^{p}(t)}{\mathrm{x}_{i}(t)} \mu_{i}\left(\mathrm{x}_{i}(t)\right)$. However, when $x_{i}^{p}(t)=0, \frac{x_{i}^{p}(t)}{\mathrm{x}_{i}(t)} \mu_{i}\left(\mathrm{x}_{i}(t)\right)=0$, since $\frac{\mu_{i}\left(\mathrm{x}_{i}(t)\right)}{\mathrm{x}_{i}(t)}$ is finite, even when $\mathrm{x}_{i}(t)=0$ given assumptions $(2 \mathrm{a})$ and $(2 \mathrm{~b})$. Thus $\dot{x}_{i}^{p}(t) \geq 0$ whenever $x_{i}^{p}(t)=0$ and we can conclude that $\boldsymbol{x}^{r}(t) \geq 0$ for all $r \in[R]$ and $t \geq 0$ and consequently $\mathbf{x}(t) \geq 0$ for all $t \geq 0$.

\subsection{Performance Control Design Objective}

The objective is to design routing parameters $\boldsymbol{\beta}(t)$ to ensure that system (4) is Positive and Conservative, and the solution $\mathbf{x}^{\beta}(t)$ satisfies the specified capacity constraints. We assume that section capacity updates would be issued at regular intervals. Thus, we assume a piecewise constant capacity constraint, as proposed, for example, in [6].

Given initial conditions and capacity constraints, a routing solution may not exist or, it may not be possible to find a feasible routing solution using the proposed control design technique. If this is the case, rather than not return a solution, we would like to return a solution which minimizes, according to some metric, the violation of the given constraint. Various metrics could be used to measure constraint violation including maximum constraint violation, average constraint violation, or total constraint violation. Here, we focus on total constraint violation for all sections throughout the entire time horizon. We introduce an upper bound $\hat{\mathbf{c}}(t)$ on the capacity constraint and minimize the integral of the difference between this upper bound and the constraint. 
Other performance metrics, such as delay or throughput, are considered secondary to the capacity constraint satisfaction and are not included in our problem formulation. Additional terms could be added to the objective function to improve system performance based on these performance metrics. This would require providing relative weighting between these performance objectives and capacity constraint violation.

The problem of interest can formally be stated as follows:

Problem 1 Given a piecewise constant vector-valued capacity constraint profile $\overline{\mathbf{c}}(t)$ on a finite time horizon $[0, H]$, find an adjusted capacity constraint $\hat{\mathbf{c}}(t) \geq \overline{\mathbf{c}}(t)$ and routing strategy $\boldsymbol{\beta}(t)$ ensuring that system (4) satisfies Positivity and Conservation and the capacity constraint condition $\mathbf{x}^{\beta}(t) \leq \hat{\mathbf{c}}(t)$, is satisfied for all $t \in[0, H]$ while minimizing $\int_{0}^{H}(\hat{\mathbf{c}}(t)-\overline{\mathbf{c}}(t)) d t$.

Here, we assume that network inflow rates and inflow routing are fixed. These values could be incorporated as control design variables, in which case additional constraints and objective terms would be required to force material through the network.

\section{Control Design}

Our previous work [1] focused on the solution of this problem for a single destination network in which the outflow rate of each section depends linearly on the amount of material in that section. This solution method can easily be extended for application to networks with multiple destinations and linear outflow rates. The control design technique presented here is based on this solution to the multiple destination, linear outflow rate problem. The significant advancement of this technique is the derivation of additional constraints and a method for generating state-dependent routing parameters for the nonlinear outflow rate system based on the routing solution for the linear outflow rate system. Together, these constraints and the routing parameter generation method ensure that the resulting routing parameters satisfy the Conservation constraints and that the state of the closed-loop nonlinear outflow rate system satisfies the capacity constraints.

In Section 3.1, the control design method of [1] is extended to the multiple destination problem with linear outflow rates. This is a relatively simple and straightforward extension, and is presented only as an intermediate step in the solution to the multiple destination nonlinear problem. The proposed solution to Problem 1 is then presented in Section 3.2.

To begin, we construct a continuous capacity bound $\mathbf{c}(t)$ in a piecewise manner using a subdivision of the time horizon of interest, $[0, H]$, into $K$ intervals of size $\Delta t$ (chosen to be a divisor of $H$ ), where $K=\frac{H}{\Delta t}$. End points of each interval of length $\Delta t$ are defined as

$$
t_{k}=k \Delta t, k=0, \ldots, K
$$

It is assumed that the capacity constraints $\overline{\mathbf{c}}(t)$ are constant over intervals of length $\Delta t$. The control design problem then becomes one of finding a routing strategy $\boldsymbol{\beta}(t)$ which ensures that $\mathbf{x}^{\beta}(t) \leq \mathbf{c}(t)$ where $\mathbf{c}(t) \leq \overline{\mathbf{c}}(t)$, when possible. If this is not possible, we would like to choose an adjusted capacity constraint $\hat{\mathbf{c}}(t)$ such that $\mathbf{c}(t) \leq \hat{\mathbf{c}}(t), \hat{\mathbf{c}}(t) \geq \overline{\mathbf{c}}(t)$ and $\int_{0}^{H}(\hat{\mathbf{c}}(t)-\overline{\mathbf{c}}(t)) d t$ is minimized.

We will be dealing with functions which may be discontinuous, with discontinuities occurring at the end points of intervals of length $\Delta t$. Therefore for any function $g$ we define

$$
g\left(t_{k}^{+}\right)=\lim _{\substack{t \rightarrow t_{k} \\ t \geq t_{k}}} g(t) \quad \text { and } \quad g\left(t_{k}^{-}\right)=\lim _{\substack{t \rightarrow t_{k} \\ t \leq t_{k}}} g(t)
$$

Constraints will be given to ensure that conditions required for Positivity, Conservation, and capacity constraint satisfaction are satisfied at the end points of each interval of length $\Delta t$, i.e. at $t_{k}, k=0, \ldots, K$. We then give a method of generating continuous time routing parameters which ensure that these conditions hold throughout the intervals.

Throughout the development of this solution method, we assume that the inflow rates $d_{s}^{r}(t)$ are piecewise constant over the intervals of length $\Delta t$ for all $r \in[R]$ and $s \in[S]$. Inflow rates which conform to this assumption may be conservatively generated from more general inflow rates by taking $d_{s}^{r}(t)$ as the maximum of the true inflow rate over the interval $\left[t_{k}, t_{k+1}\right]$ where $k=\left\lfloor\frac{t}{\Delta t}\right\rfloor$.

\subsection{Multiple Destination Linear Outflow Model}

\subsubsection{Model}

Here, we extend the solution to Problem 1 presented in [1] to address the multiple destination problem with linear outflow rates. To clearly distinguish the linear and nonlinear models, which will be used in conjunction to prove results in subsequent sections, we will introduce new notation to describe the linear outflow rate system. Sub-network state vectors $\boldsymbol{y}^{r}$, full network state vector $\mathbf{y}$, and routing parameters $\boldsymbol{\eta}$ are analogous to $\boldsymbol{x}^{r}, \mathbf{x}$, and $\boldsymbol{\beta}$, defined in Section 2.1 .

The outflow rate of section $i$ is $f_{i}\left(\mathrm{y}_{i}(t)\right)=\frac{\mathrm{y}_{i}(t)}{\tau_{i}}$ where constant $\tau_{i}>0$ is the average traversal time of section 
$i$. The dynamics of section $i$ with destination $r$ is

$$
\dot{\boldsymbol{y}}^{r}(t)=\left(\boldsymbol{\eta}^{r}(t)^{T}-\boldsymbol{I}\right) \operatorname{diag}(\boldsymbol{\tau})^{-1} \boldsymbol{y}^{r}(t)+\sum_{s=1}^{S} \boldsymbol{d}_{s}^{r}(t)
$$

Assuming a uniform distribution of material bound for each destination throughout the section, the portion of this outflow rate bound for destination $r$ is given by

$$
\frac{y_{i}^{r}(t)}{\tau_{i}} .
$$

Note that the outflow rate given above depends only on the state of section $i$ of the sub-network associated with destination $r, y_{i}^{r}(t)$. As a result, the dynamics for each destination are decoupled from all other destinations, which allows for a relatively straightforward extension of the solution of the single destination problem to the multiple destination problem. This is in contrast with the nonlinear outflow model, given in (3), which additionally depends on the full state of section $i, \mathrm{x}_{i}(t)$.

We denote the solution to (9) with initial state $\boldsymbol{y}^{r}(0)=$ $\boldsymbol{y}_{0}^{r} \geq 0$ for all $r \in[R]$ and inflow rates $d_{s}^{r}(t)$ for all $s \in$ $[S]$ and $r \in[R]$, under the particular choice of routing strategy $\boldsymbol{\eta}(t)$, as $\mathbf{y}^{\eta}(t)$.

\subsubsection{Basic Control Design Objectives}

Positivity of the newly constructed linear outflow rate system (9) is defined in the same way as it is for the general nonlinear outflow rate system (4) in Section 2.2. Note that the linear outflow rate system (9) can be generated from the more general system (4) with $\boldsymbol{\eta}$ replacing $\boldsymbol{\beta}$ and outflow rate

$$
\mu_{i}\left(\mathrm{y}_{i}(t)\right)=\frac{\mathrm{y}_{i}(t)}{\tau_{i}},
$$

which clearly satisfies the assumptions of Claim 1 . Thus, we can conclude that Positivity and Conservation hold for system (9) if $\mathcal{C}\left(\boldsymbol{\eta}^{r}(t)\right)$ hold for all $r \in[R]$.

\subsubsection{Control Design}

We now give sufficient conditions which can be used to generate a time-varying routing strategy $\boldsymbol{\eta}(t)$ ensuring that the solution to system (9) remains below a continuous capacity bound $\mathbf{c}(t)$, that is $\mathbf{y}^{\eta}(t) \leq \mathbf{c}(t)$. We focus on each sub-network separately, giving sufficient conditions to generate routing strategy $\boldsymbol{\eta}^{r}(t)$ ensuring that the state of sub-network $r$ remains below a continuous capacity bound $\boldsymbol{c}^{r}(t)$, that is $\boldsymbol{y}^{r}(t) \leq \boldsymbol{c}^{r}(t)$. We then define the capacity bound for the full network as

$$
\mathbf{c}(t)=\sum_{r=1}^{R} \boldsymbol{c}^{r}(t)
$$

These conditions are stated in the following proposition.

Proposition 2 Given a fixed value of $r \in[R]$, let $\boldsymbol{\eta}^{r}(t)$ be given such that $\eta_{i j}^{r}(t) \geq 0$ for all $i, j \in \mathcal{N}^{r}$ and $t \geq 0$ and let $t \mapsto \boldsymbol{c}^{r}(t)$ be a differentiable vector-valued map such that $\boldsymbol{c}^{r}(t)>0$ for all $t \geq 0$. Then, if $\boldsymbol{y}_{0}^{r} \leq \boldsymbol{c}^{r}(0)$ and

$$
\left(\boldsymbol{\eta}^{r}(t)^{T}-\boldsymbol{I}\right) \operatorname{diag}(\boldsymbol{\tau})^{-1} \boldsymbol{c}^{r}(t)+\sum_{s=1}^{S} \boldsymbol{d}_{s}^{r}(t) \leq \dot{\boldsymbol{c}}^{r}(t)
$$

for all $t \geq 0$ the solution of system (9) satisfies $\boldsymbol{y}^{r}(t) \leq$ $c^{r}(t)$ for all $t \geq 0$.

PROOF. The proof is similar to that of Proposition 8 of [1].

Next, we derive constraints which can be imposed at the end points of each interval and a method of interpolation of $\boldsymbol{\eta}(t)$ between these end points which ensure that the conditions needed to apply Proposition 2 hold throughout the interval.

We make the choice to use a piecewise linear capacity bound, $\boldsymbol{c}^{r}(t)$, for each destination $r \in[R]$. We parameterize this function by the initial value $\boldsymbol{c}_{0}^{r}$, and slope $\boldsymbol{m}^{r}(t) \in \mathbb{R}^{N}$, that is

$$
\boldsymbol{c}^{r}(t)= \begin{cases}\boldsymbol{c}_{0}^{r}, & t=t_{0}, \\ \boldsymbol{c}^{r}\left(t_{k-1}\right)+\Delta t \boldsymbol{m}^{r}\left(t_{k-1}^{+}\right), & t=t_{k}>t_{0}, \\ \boldsymbol{c}^{r}\left(t_{k}\right)+\left(t-t_{k}\right) \boldsymbol{m}^{r}\left(t_{k}^{+}\right), & t \neq t_{k},\end{cases}
$$

where $k=\left\lfloor\frac{t}{\Delta t}\right\rfloor$ and $\boldsymbol{m}^{r}(t)$ is constant over the intervals $\left(t_{k}, t_{k+1}\right)$ for $k=0, \ldots K-1$.

The choice of a piecewise linear capacity bound $\boldsymbol{c}^{r}(t)$ allows for a straightforward interpolation of $\boldsymbol{\eta}(t)$ between the endpoints of time intervals, while preserving the properties needed to ensure that Positivity and Conservation hold and the performance objective is satisfied. The method of interpolation is given in the following theorem.

Theorem 3 Given a fixed value of $r \in[R]$, let the capacity bound vector $\boldsymbol{c}^{r}(t)$ be given as in (10) for allt $\in[0, H]$, and $\boldsymbol{y}_{0}^{r} \leq \boldsymbol{c}_{0}^{r}$. For each $k \in\{0, \ldots, K-1\}$, if there exist $\boldsymbol{\eta}^{r}\left(t_{k}\right)$ and $\boldsymbol{\eta}^{r}\left(t_{k+1}\right)$ such that constraints $\mathcal{C}\left(\boldsymbol{\eta}^{r}\left(t_{k}\right)\right)$ and $\mathcal{C}\left(\boldsymbol{\eta}^{r}\left(t_{k+1}\right)\right)$ are satisfied and

$$
\begin{array}{r}
\left(\boldsymbol{\eta}^{r}\left(t_{k}\right)^{T}-\boldsymbol{I}\right) \operatorname{diag}(\boldsymbol{\tau})^{-1} \boldsymbol{c}^{r}\left(t_{k}\right)+\sum_{s=1}^{S} \boldsymbol{d}_{s}^{r}\left(t_{k}\right) \leq \boldsymbol{m}^{r}\left(t_{k}^{+}\right) \\
\left(\boldsymbol{\eta}^{r}\left(t_{k+1}\right)^{T}-\boldsymbol{I}\right) \operatorname{diag}(\boldsymbol{\tau})^{-1} \boldsymbol{c}^{r}\left(t_{k+1}\right)+\sum_{s=1}^{S} \boldsymbol{d}_{s}^{r}\left(t_{k+1}\right) \leq \boldsymbol{m}^{r}\left(t_{k}^{+}\right)
\end{array}
$$


then the parameters $\boldsymbol{\eta}^{r}(t)$ defined by

$$
\begin{aligned}
& \eta_{i j}^{r}(t)= \\
& \quad \frac{(1-\alpha(t)) \eta_{i j}^{r}\left(t_{k}\right) c_{i}^{r}\left(t_{k}\right)+\alpha(t) \eta_{i j}^{r}\left(t_{k+1}\right) c_{i}^{r}\left(t_{k+1}\right)}{(1-\alpha(t)) c_{i}^{r}\left(t_{k}\right)+\alpha(t) c_{i}^{r}\left(t_{k+1}\right)}
\end{aligned}
$$

for all $i, j \in \mathcal{N}^{r}$ and $t \in[0, H]$ where $k=\left\lfloor\frac{t}{\Delta t}\right\rfloor$ and $\alpha(t)=\frac{t-t_{k}}{\Delta t}$, satisfy constraints $\mathcal{C}\left(\boldsymbol{\eta}^{r}(t)\right)$ thus ensuring that System (9) is Positive and Conservative for all $t \in$ $[0, H]$. In addition, the resulting solution $\boldsymbol{y}^{r}(t)$ satisfies $\boldsymbol{y}^{r}(t) \leq \boldsymbol{c}^{r}(t)$ for all $t \in[0, H]$.

The proof of Theorem 3 is given in [1]. The proof relies on the fact that $\eta_{i j}^{r}(t)$ is a convex combination of $\eta_{i j}^{r}\left(t_{k}\right)$ and $\eta_{i j}^{r}\left(t_{k+1}\right)$ and $\eta_{i j}^{r}(t) c_{i}^{r}(t)$ is a convex combination of $\eta_{i j}^{r}\left(t_{k}\right) c_{i}^{r}\left(t_{k}\right)$ and $\eta_{i j}^{r}\left(t_{k+1}\right) c_{i}^{r}\left(t_{k+1}\right)$, and thus constraints $\mathcal{C}\left(\boldsymbol{\eta}^{r}(t)\right)$ which are linear in $\eta_{i j}^{r}(t)$, and constraints (11) which are linear in $\eta_{i j}^{r}(t) c_{i}^{r}(t)$, are satisfied for all $t \in[0, H]$ whenever they are satisfied at the end points, $t_{k}$, for $k=0, \ldots, K$. Constraints (11) can be transformed into linear constraints by introducing variable $z_{i j}^{r}(t)$ to substitute for the nonlinear terms, $\eta_{i j}^{r}(t) c_{i}^{r}(t)$, for all $i, j \in \mathcal{N}^{r}$ and all $r \in[R]$. Constraints which ensure that $\mathcal{C}\left(\boldsymbol{\eta}^{r}(t)\right)$ and Constraints (11) hold are given below, denoted by $\Phi^{r}$ :

$$
\boldsymbol{c}^{r}\left(t_{0}\right) \geq \boldsymbol{y}_{0}^{r}
$$

for all $k \in\{0, \ldots, K\}$

$$
\begin{gathered}
\boldsymbol{c}^{r}\left(t_{k}\right) \geq 0, \\
\zeta_{i j}^{r}\left(t_{k}\right) \geq 0, \forall i, j \in \mathcal{N}^{r}, \\
\zeta_{i j}^{r}\left(t_{k}\right) \leq c_{i}^{r}\left(t_{k}\right), \forall i, j \in \mathcal{N}^{r}, \\
\zeta_{i j}^{r}\left(t_{k}\right)=0, \forall j \in \mathcal{N}^{r} \backslash \mathcal{O}_{i}^{r}, \forall i \in \mathcal{N}^{r}, \\
\sum_{j=1}^{n} \zeta_{i j}^{r}\left(t_{k}\right)=c_{i}^{r}\left(t_{k}\right), \forall i \in \mathcal{N}^{r} \backslash \mathcal{S}_{\mathcal{F}}^{r}, \\
-\operatorname{diag}(\boldsymbol{\tau})^{-1} \boldsymbol{c}^{r}\left(t_{k}\right)+\boldsymbol{\zeta}^{r}\left(t_{k}\right)^{T} \operatorname{diag}(\boldsymbol{\tau})^{-1} \mathbf{1}+ \\
\qquad \sum_{s=1}^{S} \boldsymbol{d}_{s}^{r}\left(t_{k}^{+}\right) \leq \boldsymbol{m}^{r}\left(t_{k}^{+}\right), \\
\text {for all } k \in\{0, \ldots, K-1\} \\
\boldsymbol{c}^{r}\left(t_{k+1}\right)=\boldsymbol{c}^{r}\left(t_{k}\right)+\Delta t \boldsymbol{m}^{r}\left(t_{k}^{+}\right), \\
-\operatorname{diag}(\boldsymbol{\tau})^{-1} \boldsymbol{c}^{r}\left(t_{k+1}\right)+\boldsymbol{\zeta}^{r}\left(t_{k+1}\right)^{T} \operatorname{diag}(\boldsymbol{\tau})^{-1} \mathbf{1}+ \\
\quad \sum_{s=1}^{S} \boldsymbol{d}_{s}^{r}\left(t_{k}^{+}\right) \leq \boldsymbol{m}^{r}\left(t_{k}^{+}\right),
\end{gathered}
$$

For each $r \in[R]$, the values of $\boldsymbol{\eta}^{r}$ at the end points of the intervals can be recovered as

$$
\boldsymbol{\zeta}^{r}\left(t_{k}\right)^{T}=\boldsymbol{\eta}^{r}\left(t_{k}\right)^{T} \boldsymbol{c}_{r}\left(t_{k}\right)
$$

for all $k=\{0, \ldots, K\}$. These values can then be interpolated between endpoints according to (12).

A solution to Problem 1 can be generated by finding a feasible point of the linear constraints $\Phi^{r}$ for all $r \in[R]$ which also satisfies $\mathbf{c}(t) \leq \overline{\mathbf{c}}(t)$. If this problem is not feasible, we would like to give a recommendation on how to adjust capacity constraints $\overline{\mathbf{c}}(t)$ in order to find a routing solution satisfying these adjusted constraints which are, in some sense, close to the original desired constraints. For this reason, we introduce piecewise constant adjusted constraint $\hat{\mathbf{c}}(t)$. We then propose a solution to Problem 1 as finding a feasible point of $\Phi^{r}$ for all $r \in[R]$ which also satisfies $\mathbf{c}(t) \leq \hat{\mathbf{c}}(t)$ with the additional constraint $\hat{\mathbf{c}}(t) \geq \overline{\mathbf{c}}(t)$ while minimizing the integral of the difference between $\hat{\mathbf{c}}(t)$ and $\overline{\mathbf{c}}(t)$. When $\hat{\mathbf{c}}(t)=\overline{\mathbf{c}}(t)$ the given capacity constraints are satisfied.

The problem of finding capacity constraints $\hat{\mathbf{c}}$ and capacity bound $\mathbf{c}$ can then be written as the following LP problem, which will be referred to as the Routing LP Problem:

$$
\begin{aligned}
\min _{\hat{\mathbf{c}}, \mathbf{c}, \boldsymbol{\zeta}, \boldsymbol{m}} & \sum_{k=0}^{K-1} \mathbf{1}^{T}\left(\hat{\mathbf{c}}\left(t_{k}^{+}\right)-\overline{\mathbf{c}}\left(t_{k}^{+}\right)\right) \Delta t \\
\text { subject to } & \Phi^{r}, \forall r \in[R] \\
\text { for all } & k \in\{0, \ldots, K\} \\
\mathbf{c}(t) & \leq \min \left\{\hat{\mathbf{c}}\left(t_{k}^{-}\right), \hat{\mathbf{c}}\left(t_{k}^{+}\right)\right\}, \\
\hat{\mathbf{c}}\left(t_{k}^{+}\right) & \geq \overline{\mathbf{c}}\left(t_{k}^{+}\right), \\
\sum_{r=1}^{R} \boldsymbol{c}^{r}\left(t_{k}\right) & =\mathbf{c}\left(t_{k}\right) .
\end{aligned}
$$

As defined earlier, the resulting capacity bound on the total amount of material in each section is

$$
\mathbf{c}(t)=\sum_{r=1}^{R} \boldsymbol{c}^{r}(t) .
$$

Given that, under the routing strategy generated from a feasible solution of LP problem (14), the solution to system (9) satisfies $\boldsymbol{y}^{r}(t) \leq \boldsymbol{c}^{r}(t)$ for all $t \in[0, H]$, it follows that $\mathbf{y}(t) \leq \mathbf{c}(t) \leq \hat{\hat{\mathbf{c}}}(t)$ for all $t \in[0, H]$.

Note that the objective function of LP problem (14) can be any convex function of the control design variables. For instance, a different positive weight could be used for each $i$ to indicate that going over capacity in certain sections is worse than going over capacity in others. Alternatively, including the term $\mathbf{c}\left(t_{K}\right)$ in the objective function would provide an incentive for clearing material out of the network by the final time step.

\subsection{Multiple Destination Nonlinear Outflow Model}

\subsubsection{Control Design}

Here, we leverage the control design method developed in Section 3.1.3 to solve Problem 1 with general nonlinear outflow rates. The decoupled dynamics of the lin- 
ear outflow rate system allowed us to treat each subnetwork separately. However, in the nonlinear outflow rate system, outflow rates of each section of a given subnetwork depend not only on the associated state of the sub-network, but also on the full state of that section. Specifically, if we were to formulate a proposition for the nonlinear outflow rate system which parallels Proposition 2 for the linear outflow rate system, the proof would require us to lower bound the term

$$
\frac{c_{j}^{r}(t)}{\mathrm{c}_{j}(t)} \mu_{j}\left(\mathrm{c}_{j}(t)\right)-\frac{x_{j}^{r}(t)}{\mathrm{x}_{j}(t)} \mu_{j}\left(\mathrm{x}_{j}(t)\right) .
$$

Unfortunately, we have no way to bound this term within the given framework.

Rather than solving the nonlinear outflow rate problem directly, we take the solution method for the linear outflow problem and add additional constraints on the routing parameters which force recirculation within each section of the network. This recirculation effectively slows down the linear outflow rate system so that the controlled outflow rate of each section of the linear outflow rate system is at or below the corresponding uncontrolled nonlinear outflow rate. These conditions are formally stated in Theorem 4 below.

Theorem 4 Let $\boldsymbol{c}^{r}(t)$ be defined as in (10) with $\boldsymbol{m}^{r}\left(t_{k}^{+}\right)$ for $k=0, \ldots, K$ and $\boldsymbol{c}_{0}^{r} \geq x_{0}^{r}$ given for all $r \in[R]$. Let an outflow function $\mu$ satisfying assumptions (2) be given. Define $\tau_{i}=\left(\frac{d \mu_{i}}{d \mathrm{x}_{i}}(0)\right)^{-1}$ for all $i \in[N]$. If, for all $r \in[R]$, there exist $\boldsymbol{\eta}^{r}\left(t_{k}\right)$ for $k=0, \ldots, K$, such that constraints $\mathcal{C}\left(\boldsymbol{\eta}^{r}\left(t_{k}\right)\right)$ are satisfied and the following constraints hold for $k=0, \ldots K-1$

$$
\begin{array}{r}
\left(\boldsymbol{\eta}^{r}\left(t_{k}\right)^{T}-\boldsymbol{I}\right) \operatorname{diag}(\boldsymbol{\tau})^{-1} \boldsymbol{c}^{r}\left(t_{k}\right)+\sum_{s=1}^{S} \boldsymbol{d}_{s}^{r}\left(t_{k}^{+}\right) \leq \boldsymbol{m}^{r}\left(t_{k}^{+}\right) \\
\left(\boldsymbol{\eta}^{r}\left(t_{k+1}\right)^{T}-\boldsymbol{I}\right) \operatorname{diag}(\boldsymbol{\tau})^{-1} \boldsymbol{c}^{r}\left(t_{k+1}\right)+\sum_{s=1}^{S} \boldsymbol{d}_{s}^{r}\left(t_{k}^{+}\right) \leq \boldsymbol{m}^{r}\left(t_{k}^{+}\right)
\end{array}
$$

$$
\begin{aligned}
\eta_{i i}^{r}\left(t_{k}\right) & \geq \underline{\eta}_{i}(k), \forall i \in \mathcal{N}^{r}, \\
\eta_{i i}^{r}\left(t_{k+1}\right) & \geq \underline{\eta}_{i}(k), \forall i \in \mathcal{N}^{r},
\end{aligned}
$$

where the fixed scalar value $\underline{\eta}_{i}(k)$ is chosen such that

$$
\underline{\eta}_{i}(k) \geq \max _{t_{k} \leq t \leq t_{k+1}}\left(1-\frac{\tau_{i} \mu_{i}\left(\mathrm{c}_{i}(t)\right)}{\mathrm{c}_{i}(t)}\right),
$$

for each $i \in N$ and $k \in[0, \ldots, K-1]$, then the closed-loop system (4) under the decentralized time-varying state feedback control policy

$$
\boldsymbol{\beta}^{r}(t)^{T}=\boldsymbol{I}+\left(\boldsymbol{\eta}^{r}(t)^{T}-\boldsymbol{I}\right) \operatorname{diag}(\mathbf{x}(t)) \operatorname{diag}(\boldsymbol{\tau})^{-1} \operatorname{diag}(\boldsymbol{\mu}(\mathbf{x}(t)))^{-1}
$$

with $\boldsymbol{\eta}^{r}(t)$ interpolated as in (12), has the following properties

(i) The solution $\mathbf{x}^{\beta}(t)$ of system (4) is identical to the solution $\mathbf{y}^{\eta}(t)$ of system (9) with routing parameters $\boldsymbol{\eta}(t)$

(ii) Positivity holds for system (4),

(iii) Conservation holds for system (4),

(iv) The solution $\mathbf{x}^{\beta}(t)$ of system (4), satisfies $\mathbf{x}^{\beta}(t) \leq$ $\mathbf{c}(t)$ for $t \in[0, H]$.

PROOF. We first note that the feedback control policy defined by (17) was chosen so that the nonlinear outflow rate system (4) in closed-loop is identical to the linear outflow rate system (9) with routing parameters $\boldsymbol{\eta}(t)$. To see this, we substitute the expressions for $\boldsymbol{\beta}(t)$ given in (17) into (4), which describes the dynamics of each section $i \in \mathcal{N}^{r}$ of the sub-network associated with destination $r \in[R]$. This simplifies to

$$
\dot{\boldsymbol{x}}^{r}(t)=\left(\boldsymbol{\eta}^{r}(t)^{T}-\boldsymbol{I}\right) \operatorname{diag}(\boldsymbol{\tau})^{-1} \boldsymbol{x}^{r}(t)+\sum_{s=1}^{S} \boldsymbol{d}_{s}^{r}(t)
$$

which is the same as the description of the dynamics of sub-network $r \in[R]$ of the linear outflow rate system given in (9). Thus, if $\boldsymbol{y}^{r}(0)=\boldsymbol{x}^{r}(0)=\boldsymbol{x}_{0}^{r}$ for all $r \in[R]$ then $\boldsymbol{x}^{r}(t)=\boldsymbol{y}^{r}(t)$ and $(i)$ holds. Additionally, since inequalities (15) are the same as those in (11), and $\boldsymbol{\eta}^{r}(t)$ is interpolated between the endpoints $t_{k}$ of each interval by (12), for $k=0, \ldots K$, Theorem 3 can be applied to conclude that Positivity holds for system (9), that is $\boldsymbol{y}^{r}(t) \geq 0$, for all $r \in[R]$, and $\mathbf{y}(t) \leq \mathbf{c}(t)$, for $t \in[0, H]$. Given $(i)$, these properties also hold for system (4), thus (ii) and (iv) hold.

Finally, we will show that Conservation holds by verifying that $\mathcal{C}\left(\boldsymbol{\beta}^{r}(t)\right)$ hold for all $r \in[R]$. From Theorem 3, we know that constraints $\mathcal{C}\left(\boldsymbol{\eta}^{r}(t)\right)$ hold for all $r \in[R]$. It is easily seen that $\mathcal{C}_{2}\left(\boldsymbol{\beta}^{r}(t)\right)$ holds whenever $\mathcal{C}_{2}\left(\boldsymbol{\eta}^{r}(t)\right)$ holds. To check $\mathcal{C}_{3}\left(\boldsymbol{\beta}^{r}(t)\right)$ and $\mathcal{C}_{4}\left(\boldsymbol{\beta}^{r}(t)\right)$ we compute $\boldsymbol{\beta}^{r}(t)$ 1. We first introduce a diagonal matrix consisting of the ratios of the linear outflow rate and non-linear outflow rate for each section

$$
\mathcal{F}(\mathbf{x}(t))=\operatorname{diag}(\mathbf{x}(t)) \operatorname{diag}(\boldsymbol{\tau})^{-1} \operatorname{diag}(\boldsymbol{\mu}(\mathbf{x}(t)))^{-1} .
$$

Then

$$
\begin{aligned}
\boldsymbol{\beta}^{r}(t) \mathbf{1} & =\left[\boldsymbol{I}+\left(\boldsymbol{\eta}^{r}(t)^{T}-\boldsymbol{I}\right) \mathcal{F}(\mathbf{x}(t))\right]^{T} \mathbf{1} \\
& =1+\left[\mathcal{F}(\mathbf{x}(t))\left(\boldsymbol{\eta}^{r}(t)-\boldsymbol{I}\right)\right] \mathbf{1} .
\end{aligned}
$$

Given that constraints $\mathcal{C}_{3}\left(\boldsymbol{\eta}^{r}(t)\right)$ are satisfied, that is 
we can conclude from (18) that constraints $\mathcal{C}_{3}\left(\boldsymbol{\beta}^{r}(t)\right)$ hold, that is

$$
\sum_{j \in \mathcal{O}_{i}^{r}} \beta_{i j}^{r}(t)=1, \forall i \in \mathcal{N}^{r} \backslash \mathcal{S}_{\mathcal{F}}^{r}
$$

And given that constraints $\mathcal{C}_{4}\left(\boldsymbol{\eta}^{r}(t)\right)$ are satisfied, that is

$$
\sum_{j \in \mathcal{O}_{i}^{r}} \eta_{i j}^{r}(t) \leq 1, \forall i \in \mathcal{S}_{\mathcal{F}}^{r}
$$

we can conclude from (18) that constraints $\mathcal{C}_{4}\left(\boldsymbol{\beta}^{r}(t)\right)$ hold, that is

$$
\sum_{j \in \mathcal{O}_{i}^{r}} \beta_{i j}^{r}(t) \leq 1, \forall i \in \mathcal{S}_{\mathcal{F}}^{r}
$$

Clearly constraint $\mathcal{C}_{1}\left(\boldsymbol{\beta}^{r}(t)\right)$ holds when $i \neq j$ for all $r \in[R]$. In order to show that $\mathcal{C}_{1}\left(\boldsymbol{\beta}^{r}(t)\right)$ holds when $i=j$, we must show that

$$
\eta_{i i}^{r}(t) \geq 1-\frac{\tau_{i} \mu_{i}\left(\mathrm{x}_{i}(t)\right)}{\mathrm{x}_{i}(t)},
$$

for all $i \in \mathcal{N}^{r}, r \in[R], t \in[0, H]$. First, we show that

$$
\frac{\mu_{i}\left(\mathrm{x}_{i}(t)\right)}{\mathrm{x}_{i}(t)} \geq \frac{\mu_{i}\left(\mathrm{c}_{i}(t)\right)}{\mathrm{c}_{i}(t)}
$$

whenever $\mathrm{x}_{i}(t) \leq \mathrm{c}_{i}(t)$ and thus

$$
\eta_{i i}^{r}(t) \geq 1-\frac{\tau_{i} \mu_{i}\left(\mathrm{c}_{i}(t)\right)}{\mathrm{c}_{i}(t)},
$$

for all $i \in \mathcal{N}^{r}, r \in[R], t \in[0, H]$ implies that (19) holds. In order to show $(20)$ we use the facts that $\mu_{i}$ is concave, $\mu_{i}(0)=0,0 \leq \mathrm{x}_{i}(t) \leq \mathrm{c}_{i}(t)$ which follows from $(i i)$ and $(i v)$, and $\mathrm{x}_{i}(t)=\frac{\mathrm{x}_{i}(t)}{\mathrm{c}_{i}(t)} c_{i}(t)+\left(1-\frac{\mathrm{x}_{i}(t)}{c_{i}(t)}\right) \times 0$, therefore

$$
\begin{aligned}
\mu_{i}\left(\mathrm{x}_{i}(t)\right) & \geq \frac{\mathrm{x}_{i}(t)}{\mathrm{c}_{i}(t)} \mu_{i}\left(\mathrm{c}_{i}(t)\right)+\left(1-\frac{\mathrm{x}_{i}(t)}{\mathrm{c}_{i}(t)}\right) \mu_{i}(0) \\
\mu_{i}\left(\mathrm{x}_{i}(t)\right) & \geq \frac{\mathrm{x}_{i}(t)}{\mathrm{c}_{i}(t)} \mu_{i}\left(\mathrm{c}_{i}(t)\right) \\
\frac{\mu_{i}\left(\mathrm{x}_{i}(t)\right)}{\mathrm{x}_{i}(t)} & \geq \frac{\mu_{i}\left(\mathrm{c}_{i}(t)\right)}{\mathrm{c}_{i}(t)} .
\end{aligned}
$$

From (12) and the fact that $c_{i}^{r}(t)$ is piecewise linear, we have

$$
\begin{aligned}
\eta_{i i}^{r}(t) c_{i}^{r}(t)= & \left(1-\frac{t-t_{k}}{\Delta t}\right) \eta_{i i}^{r}\left(t_{k}\right) c_{i}^{r}\left(t_{k}\right) \\
& +\frac{t-t_{k}}{\Delta t} \eta_{i i}^{r}\left(t_{k+1}\right) c_{i}^{r}\left(t_{k+1}\right) \\
\geq & c_{i}^{r}(t) \underline{\eta}_{i}(k)
\end{aligned}
$$

where the inequality follows from constraints (16). Thus, $\eta_{i i}^{r}(t) \geq \underline{\eta}_{i}(k)$ where $k=\left\lfloor\frac{t}{\Delta t}\right\rfloor$. Given the definition of $\underline{\eta}_{i}(k)$, it follows that (21) and thus (19) hold and consequently that (5) holds for $\beta_{i i}^{r}(t)$ defined as in (17).

Since constraints $\mathcal{C}\left(\boldsymbol{\beta}^{r}(t)\right)$ hold for all $r \in[R]$, we can conclude that Conservation holds for system (4) in closed-loop, thus (iii) holds.

The idea motivating the development of Theorem 4 is that the outflow rates of each section of linear outflow rate system (9) with $\tau_{i}=\left(\frac{d \mu_{i}}{d \mathrm{x}_{i}}(0)\right)^{-1}$ for all $i \in[N]$ can be restricted through constraints on recirculation so that the linear outflow rate system has controlled outflow rate less than the corresponding uncontrolled nonlinear outflow rate, that is

$$
\sum_{r=1}^{R}\left(1-\eta_{i i}^{r}(t)\right) \frac{y_{i}^{r}(t)}{\tau_{i}} \leq \mu_{i}\left(\mathrm{y}_{i}(t)\right)
$$

where the left hand side is the total controlled outflow rate of section $i$ of the linear outflow rate system. Constraints (16) ensure that (22) is satisfied. Given that $\underline{\eta}_{i}(k)$ is chosen based on the upper bound $\mathrm{c}_{i}(t)$ of the state $\mathrm{y}_{i}(t)$ itself, these constraints are conservative. Recirculation beyond that required to satisfy constraints (16) may be used to satisfy the control design objective. Additional recirculation in the linear outflow rate system beyond that required to satisfy (22) with equality is translated into recirculation in the controlled nonlinear outflow rate system.

It is important to note that the routing strategy defined by (17) is conservative. This is due to the fact that the conditions of Theorem 4 depend on the piecewise linear, continuous, upper bound of the state, $\mathbf{c}(t)$, rather than the state itself. In the limit as the time step $\Delta t$ is decreased to zero, (15) and (16) can hold with equality and $\mathbf{c}(t)$ approaches $\mathbf{x}(t)$.

We would like to write an LP problem, similar to LP problem (14), which can be used to generate parameters which satisfy the assumptions of Theorem 4 . Constraints (15) and $\mathcal{C}\left(\boldsymbol{\eta}^{r}\left(t_{k}\right)\right)$ for $k=0, \ldots, K-1$ are ensured by constraints $\Phi^{r}$. The constraints

$$
\begin{aligned}
\zeta_{i i}^{r}\left(t_{k}\right) & \geq \underline{\eta}_{i}(k) c_{i}^{r}\left(t_{k}\right) \\
\zeta_{i i}^{r}\left(t_{k+1}\right) & \geq \underline{\eta}_{i}(k) c_{i}^{r}\left(t_{k+1}\right)
\end{aligned}
$$

will ensure that constraints (16) hold.

What remains is to find an appropriate choice of $\underline{\eta}_{i}(k)$ for all $i \in[N]$ and $k=0, \ldots, K$. If a piecewise linear 
upper bound $\tilde{\mathbf{c}}(t)$ on $\mathbf{c}(t)$ were known, we could define the desired lower bound on recirculation parameter $\eta_{i i}^{r}(t)$ as

$$
\underline{\eta}_{i}(k)=\max _{t_{k} \leq s \leq t_{k+1}}\left(1-\tau_{i} \frac{\mu_{i}\left(\tilde{\mathrm{c}}_{i}(s)\right)}{\tilde{\mathrm{c}}_{i}(s)}\right)
$$

where $k=\left\lfloor\frac{t}{\Delta t}\right\rfloor$. Given the fact that $\tilde{\mathrm{c}}_{i}(t)$ is defined to be linear between $t_{k}$ and $t_{k+1}$ for $k \in[0, \ldots, K-1]$, and thus

$$
\tilde{\mathrm{c}}_{i}(t) \leq \max _{s \in\left\{t_{k}, t_{k+1}\right\}} \tilde{\mathrm{c}}_{i}(s)
$$

we could then proceed as in (20) to conclude that the expression in the right hand side of (23) achieves its maximum value at an end point of the interval. Since $\mathbf{c}$ is a design variable, we do not have an a priori upper bound available. Instead, we explicitly introduce $\tilde{\mathbf{c}}$, use it to define $\underline{\boldsymbol{\eta}}$ and add constraints to ensure that $\mathbf{c}(t) \leq \tilde{\mathbf{c}}(t)$.

The problem of finding capacity constraints $\hat{\mathbf{c}}$ and capacity bound $\mathbf{c}$ can then be written as the following LP problem, which will be referred to as the Routing $L P$ Problem:

$$
\min _{\hat{\mathbf{c}}, \mathbf{c}, \boldsymbol{\zeta}, \boldsymbol{m}} \sum_{k=0}^{K-1} \mathbf{1}^{T}\left(\hat{\mathbf{c}}\left(t_{k}^{+}\right)-\overline{\mathbf{c}}\left(t_{k}^{+}\right)\right) \Delta t
$$

subject to $\Phi^{r}$

$$
\begin{aligned}
\text { for all } k & \in\{0, \ldots, K\} \\
\mathbf{c}\left(t_{k}\right) & \leq \min \left\{\hat{\mathbf{c}}\left(t_{k}^{-}\right), \hat{\mathbf{c}}\left(t_{k}^{+}\right)\right\}, \\
\hat{\mathbf{c}}\left(t_{k}^{+}\right) & \geq \overline{\mathbf{c}}\left(t_{k}^{+}\right), \\
\sum_{r=1}^{R} \boldsymbol{c}^{r}\left(t_{k}\right) & =\mathbf{c}\left(t_{k}\right), \\
\mathbf{c}\left(t_{k}\right) & \leq \tilde{\mathbf{c}}\left(t_{k}\right), \\
z_{i i}^{r}\left(t_{k}\right) & \geq \underline{\eta}_{i}(k) c_{i}^{r}\left(t_{k}\right), \forall i \in \mathcal{N}^{r}, \forall r \in[R] .
\end{aligned}
$$

The specific choice of $\tilde{\mathbf{c}}(t)$ is important. If $\tilde{\mathbf{c}}(t)$ is chosen too high, then $\eta_{i i}^{r}(t)$ is forced to be high, resulting in a solution requiring more recirculation than necessary. If $\tilde{\mathbf{c}}(t)$ is too low, the Routing LP Problem (24) may be infeasible. In Section 3.2.2 an algorithm is presented in which $\tilde{\mathbf{c}}(t)$ is adjusted iteratively allowing an arbitrarily tight upper bound on $\mathbf{c}(t)$ to be converged upon.

\subsubsection{Iterative Solution Method}

Here we present Algorithm 1 which can be used to solve Problem 1 by successively solving instances of the Routing LP Problem (24). At the beginning of the algorithm, the upper bound $\tilde{\mathbf{c}}(t)$ on the capacity bound $\mathbf{c}(t)$ is set infinitely high, which forces full recirculation, that is $\eta_{i}^{r}\left(t_{k}\right)=1$ for all $i \in[N], r \in[R]$ and $k \in[0, \ldots, K-1]$. After the Routing LP Problem (24) is solved, an appropriate finite value of $\tilde{\mathbf{c}}(t)$ can be chosen. At each subsequent iteration, $\tilde{\mathbf{c}}(t)$ is decreased and, correspondingly, required recirculation is also decreased.
The algorithm terminates when the maximum difference between $\tilde{\mathbf{c}}(t)$ and $\mathbf{c}(t)$ falls below some specified threshold. This termination condition ensures that the imposed recirculation constraints are reasonable with respect to the recirculation constraints required based on the resulting capacity bound $\mathbf{c}(t)$.

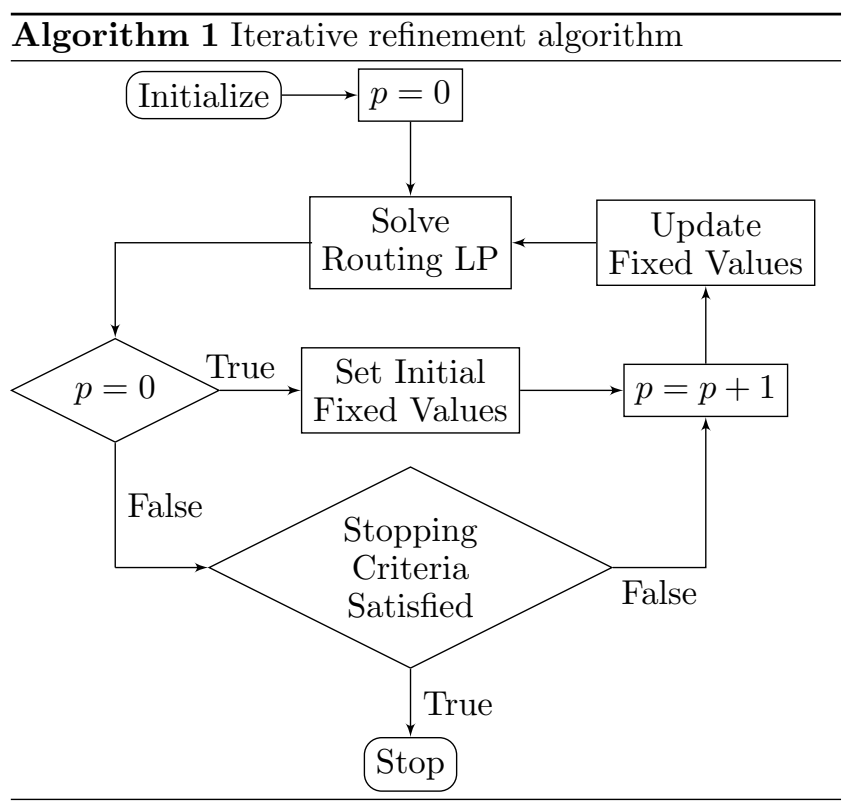

Details of the steps carried out in each block of Algorithm 1 are given in Table 2 .

User defined values $\varepsilon>0$ and $0<\gamma<1$ affect the termination and speed of convergence of the algorithm, respectively. We define $y^{\max }$ as the greatest amount of material that could occupy any section in the network by

$$
\mathrm{y}^{\max }=\sum_{r=1}^{R} \mathbf{1}^{T} \boldsymbol{y}_{0}^{r}+\sum_{r=1}^{R} \sum_{s=1}^{S} \sum_{k=0}^{K-1} \boldsymbol{d}_{s}^{r}\left(t_{k}^{+}\right) \Delta t
$$

In the following proposition, we show that Algorithm 1 is an effective method, that is, it terminates and is consistent.

Proposition 5 Given a network described as in (4) with section outflow rates $\mu$ satisfying assumptions (2) and a piecewise constant desired capacity constraint $\overline{\mathbf{c}}(t)$ for $t \in[0, H], \varepsilon>0$ and $0<\gamma<1$, the following properties hold

(i) The Routing LP Problem (24) is feasible when solved after the initialization step of Algorithm 1,

(ii) The Routing LP Problem (24) is feasible when solved at each subsequent iteration of Algorithm 1,

(iii) Algorithm 1 terminates.

PROOF. First, we show that the Routing LP Problem (24) is feasible when solved after the initialization step. 
Table 2

Details of steps of Algorithm 1.

$$
\begin{aligned}
\text { Initialize: } & \\
\text { for } k & =0, \ldots, K \\
\tilde{\mathbf{c}}\left(t_{k}\right) & =\infty \\
\underline{\boldsymbol{\eta}}(k) & =1
\end{aligned}
$$

\section{Solve LP problem}

Solve the Routing LP Problem (24)

$$
\mathbf{c}^{(p)}\left(t_{k}\right)=\mathbf{c}\left(t_{k}\right), \forall k
$$
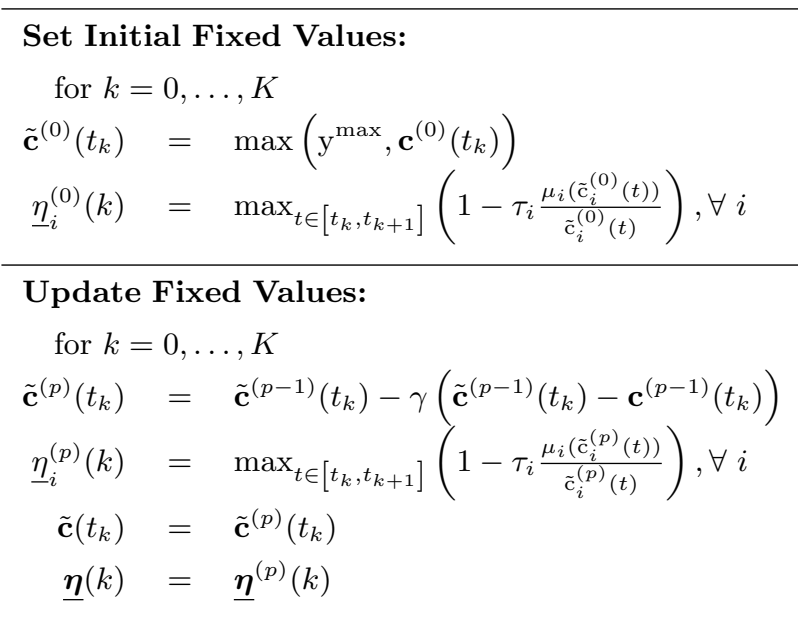

\section{Stopping Criteria:}

$\tilde{\mathbf{c}}^{(p)}\left(t_{k}\right) \quad-\quad \mathbf{c}^{(p)}\left(t_{k}\right) \leq \varepsilon$, for $k=0, \ldots, K$

At the first iteration $\eta_{i}(k)=1$ for all $i \in[N]$ and $k \in$ $[0, \ldots, K-1]$. This implies recirculation of all of the outflow of each section of the network. To construct a feasible solution, make the choice of:

$$
\begin{aligned}
\zeta_{i j}^{r}\left(t_{k}\right) & =\left\{\begin{array}{l}
c_{i}^{r}\left(t_{k}\right), i=j \\
0, j \neq i
\end{array}\right. \\
m_{i}^{r}\left(t_{k}^{+}\right) & =\max \left(\sum_{s=1}^{S} d_{s i}^{r}\left(t_{k}^{+}\right), \sum_{s=1}^{S} d_{s i}^{r}\left(t_{k+1}^{-}\right)\right)
\end{aligned}
$$

for all $i, j \in \mathcal{N}^{r}, r \in[R]$, and $k \in[0, \ldots, K-1]$. Choose $\boldsymbol{c}^{r}\left(t_{0}\right) \geq \boldsymbol{x}_{0}^{r}$ for each $r \in[R]$ and feasible values for $\boldsymbol{c}^{r}\left(t_{k}\right)$ for all $r \in[R]$ and $k \in[0, \ldots, K-1]$ can be generated using (10) given $\boldsymbol{m}^{r}\left(t_{k}\right)$. It is then straightforward to verify that constraints $\Phi^{r}$ are satisfied for all $r \in[R]$. Given that initially, $\tilde{\mathbf{c}}\left(t_{k}\right)=\infty$ for all $k \in[0, \ldots, K-1]$ it is easily seen that constraints (24b) through (24f) also hold, and thus the Routing LP Problem is feasible.

Given a feasible solution to the Routing LP Problem (24) at iteration $p-1$ for $p>0$, we show that this solution is also feasible at iteration $p$. At each iteration of Algorithm 1, $\tilde{\mathbf{c}}$ and $\boldsymbol{\eta}$ are the only constraint parameters of the Routing LP Problem (24) which change. In order to show that the Routing LP Problem (24) is feasible at each iteration, we show that the updated values of $\tilde{\mathbf{c}}$ and $\boldsymbol{\eta}$ lead to a relaxation of constraints in the LP problem compared to the previous iteration. Parameters $\tilde{\mathbf{c}}$ and $\boldsymbol{\eta}$ appear in only one constraint each, that is, constraint (24e) and constraint (24f), respectively.

We begin by focusing on parameter $\tilde{\mathbf{c}}$ and constraint $(24 \mathrm{e})$. For all $p>0$, we have

$$
\begin{aligned}
\mathbf{c}^{(p-1)}\left(t_{k}\right) & =\tilde{\mathbf{c}}^{(p-1)}\left(t_{k}\right)-\left(\tilde{\mathbf{c}}^{(p-1)}\left(t_{k}\right)-\mathbf{c}^{(p-1)}\left(t_{k}\right)\right) \\
& \leq \tilde{\mathbf{c}}^{(p-1)}\left(t_{k}\right)-\gamma\left(\tilde{\mathbf{c}}^{(p-1)}\left(t_{k}\right)-\mathbf{c}^{(p-1)}\left(t_{k}\right)\right) \\
& =\tilde{\mathbf{c}}^{(p)}\left(t_{k}\right) .
\end{aligned}
$$

The inequality follows from the fact that $\mathbf{c}^{(p-1)}\left(t_{k}\right) \leq$ $\tilde{\mathbf{c}}^{(p-1)}\left(t_{k}\right)$ given that $\mathbf{c}^{(p-1)}$ is feasible for the Routing LP Problem (24) at iteration $p-1$ and $\gamma<1$. Thus, any c feasible at iteration $p-1$ satisfies $(24 \mathrm{e})$ at iteration $p$.

We now focus on parameter $\boldsymbol{\eta}$ and constraint (24f). Note that, for any feasible solution of the Routing LP Problem $(24), \mathbf{c}^{(p-1)}\left(t_{k}\right) \leq \tilde{\mathbf{c}}^{(p-1)}\left(t_{k}\right)$. Given $0<\gamma<1$, it follows from the definition of $\tilde{\mathbf{c}}^{(p-1)}$ given in step 2 of Algorithm 1 that $\tilde{\mathbf{c}}^{(p)}\left(t_{k}\right) \leq \tilde{\mathbf{c}}^{(p-1)}\left(t_{k}\right)$. Also note that constraints $\Phi^{r}$ ensure that $\mathbf{c}\left(t_{k}\right)=\sum_{r=1}^{R} \boldsymbol{c}^{r}\left(t_{k}\right) \geq 0$, and constraint (24e) ensures that $\tilde{\mathbf{c}}\left(t_{k}\right) \geq \mathbf{c}\left(t_{k}\right)$, thus $\tilde{\mathbf{c}}^{(p)}\left(t_{k}\right) \geq 0$. Given this, the fact that $\mu_{i}$ is concave, $\mu_{i}(0)=0$ and that

$$
\frac{\mu_{i}\left(\tilde{\mathrm{c}}_{i}^{(p)}\left(t_{k}\right)\right)}{\tilde{\mathrm{c}}_{i}^{(p)}\left(t_{k}\right)} \geq \frac{\mu_{i}\left(\tilde{\mathrm{c}}_{i}^{(p-1)}\left(t_{k}\right)\right)}{\tilde{\mathrm{c}}_{i}^{(p-1)}\left(t_{k}\right)}
$$

whenever $\tilde{\mathrm{c}}_{i}^{(p)}\left(t_{k}\right) \leq \tilde{\mathrm{c}}_{i}^{(p-1)}\left(t_{k}\right)$, as shown in the proof of Theorem 4, we have,

$$
\begin{aligned}
\underline{\eta}_{i}^{(p)}(k) & =1-\frac{\tau_{i} \mu_{i}\left(\tilde{\mathrm{c}}_{i}^{(p)}\left(t_{k}\right)\right)}{\tilde{\mathrm{c}}_{i}^{(p)}\left(t_{k}\right)} \\
& \leq 1-\frac{\tau_{i} \mu_{i}\left(\tilde{\mathrm{c}}_{i}^{(p-1)}\left(t_{k}\right)\right)}{\tilde{\mathrm{c}}_{i}^{(p-1)}\left(t_{k}\right)} \\
& =\underline{\eta}_{i}^{(p-1)}(k) .
\end{aligned}
$$

It follows that constraint (24f) can be satisfied at iteration $p$ by $\boldsymbol{\zeta}$ and $\mathbf{c}$ which are feasible at iteration $p-1$, recognizing that any feasible $\boldsymbol{\zeta}$ and $\mathbf{c}$ are non-negative. Thus, we can conclude that any feasible solution of the Routing LP Problem (24) at iteration $p-1, p>0$, is also a feasible solution of the LP problem at iteration $p$ and consequently $(i i)$ holds.

We now show that Algorithm 1 is guaranteed to terminate. We focus on section $i$, time step $t_{k}$. From a rearrangement of the assignment of $\tilde{\mathbf{c}}^{(p)}\left(t_{k}\right)$ in step 2, we 
have

$$
\tilde{\mathbf{c}}^{(p-1)}\left(t_{k}\right)-\tilde{\mathbf{c}}^{(p)}\left(t_{k}\right)=\gamma\left(\tilde{\mathbf{c}}^{(p-1)}\left(t_{k}\right)-\mathbf{c}^{(p-1)}\left(t_{k}\right)\right) .
$$

Constraints $\Phi^{r}$ ensure that $\mathbf{c}^{(p-1)}\left(t_{k}\right) \leq \tilde{\mathbf{c}}^{(p-1)}\left(t_{k}\right)$, thus the right hand side of the expression above is nonnegative and the sequence $\left(\tilde{\mathrm{c}}_{i}^{(p)}\left(t_{k}\right)\right)_{p \in \mathbb{N}}$ is non-increasing. This sequence is bounded below since constraints $\Phi^{r}$ ensure that $\mathbf{c}\left(t_{k}\right)=\sum_{r=1}^{R} \boldsymbol{c}^{r}\left(t_{k}\right) \geq 0$, and constraint $(24 \mathrm{e})$ ensures that $\tilde{\mathbf{c}}\left(t_{k}\right) \geq \mathbf{c}\left(t_{k}\right)$. Since sequence $\left(\tilde{\mathrm{c}}_{i}^{(p)}\left(t_{k}\right)\right)_{p \in \mathbb{N}}$ is monotone and bounded, it converges. Any convergent sequence is a Cauchy sequence and there thus exists an integer $P_{i k}$ such that for $p \geq P_{i k}$

$$
\left|\tilde{\mathrm{c}}_{i}^{(p-1)}\left(t_{k}\right)-\tilde{\mathrm{c}}_{i}^{(p)}\left(t_{k}\right)\right|<\gamma \varepsilon
$$

Substituting the expression for $\tilde{\mathrm{c}}_{i}^{(p-1)}\left(t_{k}\right)-\tilde{\mathrm{c}}_{i}^{(p)}\left(t_{k}\right)$ given in (25) we have

$$
\left|\tilde{\mathrm{c}}_{i}^{(p-1)}\left(t_{k}\right)-\mathrm{c}_{i}^{(p-1)}\left(t_{k}\right)\right|<\varepsilon .
$$

It follows that for large enough values of $p$ (i.e. $p \geq$ $\left.\max _{i, k} P_{i k}\right)$, the stopping criteria will be satisfied and Algorithm 1 will terminate.

Note that at each iteration of Algorithm 1, constraints are relaxed compared to the previous iteration. Thus, the cost of the Routing LP Problem (24) decreases or remains constant from one iteration to the next. Physically, this means that the capacity constraint violation of the resulting closed-loop system decreases or remains constant at each iteration.

Upon termination of Algorithm 1 (and, in fact, at any iteration of the algorithm), routing parameters $\boldsymbol{\eta}\left(t_{k}\right)$ and $\boldsymbol{\eta}(t)$ can be recovered from (13) and (12), respectively. Under the decentralized time-varying state feedback control policy defined by (17), system (4) satisfies the Positivity and Conservation constraints, and the solution $\mathbf{x}^{\beta}(t)$ to system (4) satisfies $\mathbf{x}^{\beta}(t) \leq \mathbf{c}(t) \leq \hat{\mathbf{c}}(t)$ for all $t \in[0, H]$.

\section{Application}

In order to illustrate the control design technique presented in Section 3, this technique was applied to an air traffic control example problem. The network used in this problem is shown in Figure 1 presented in Section 2.1. Network connectivity for the full network and subnetworks associated with destinations 1, 2 and 3 can be inferred from Figures 1(a), 1(b), 1(c), and 1(d), respectively. Note that, although not explicitly depicted, recirculation is allowed in all sections of the network.

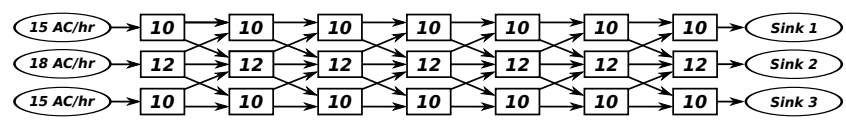

(a) Full network.

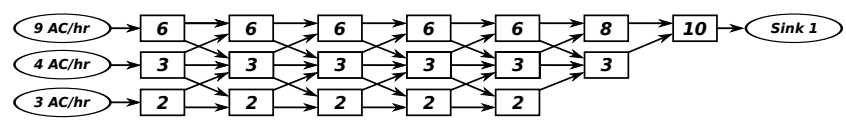

(b) Sub-network for destination 1.

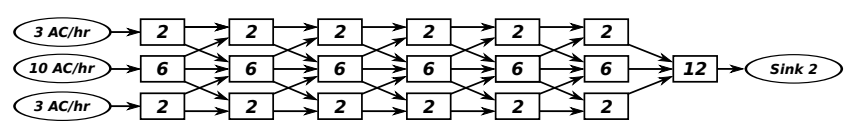

(c) Sub-network for destination 2.

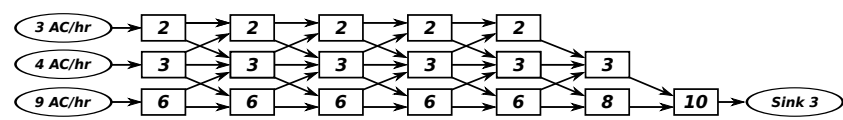

(d) Sub-network for destination 3.

Fig. 2. Figures 2(a), 2(b), 2(c), and 2(d) indicate inflow rates and section initial conditions for all flights in the network and flights with destination 1,2 and 3 , respectively. The inflow at sources is indicated in the ovals on the left of each diagram. The value in each box represents the initial number of aircraft in that section.

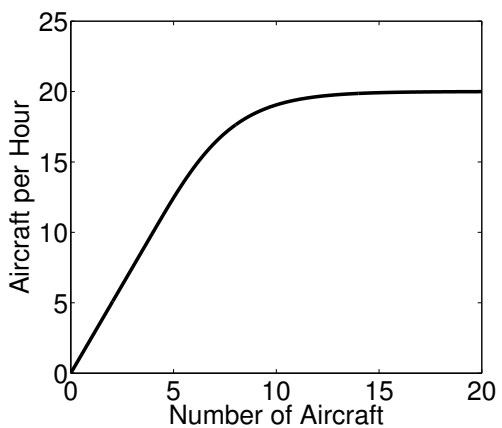

Fig. 3. Section outflow rate as a function of the total number of aircraft in the section. The same outflow rate function is used for each section of the network.

Initial conditions and inflow rates are depicted graphically in Figure 2. Inflow rates are constant for the duration of the planning horizon of 3 hours. Flow rates and initial states are broken down by destination in Figures 2(b), 2(c) and 2(d). That is, in Figure 2(b), the inflow rates specified are the inflow rates of flights with destination 1 , that is, $d_{s i}^{1}(t)$ for each source, $s=1,2,3$ and each section accepting inflow, $i=1,2,3$. The values in the boxes in figure Figure 2(b) indicate the initial number of aircraft in each section with destination 1 , that is, mathematically, $x_{i}^{1}(0)$ for all $i \in \mathcal{N}^{1}$.

The outflow rate, $\mu_{i}$, as a function of the number of aircraft in the section is given in Figure 3. This outflow rate function is used for all sections of the network. For 
each section $i \in[N]$,

$$
\tau_{i}=\left(\frac{d \mu_{i}}{d \mathrm{x}_{i}}(0)\right)^{-1}=0.4 \text { hours }
$$

Each section, except for section 14 , has a constant capacity constraint of 15 aircraft, that is, $\bar{c}_{i}(t)=15$ aircraft for all $i \in[N] \backslash\{14\}$ and all $t \geq 0$. Section 14 has the piecewise constant capacity constraint profile $\bar{c}_{14}(t)$ pictured in Figure 4(a).

Given the specified initial conditions, inflow rate and outflow rate function, if the outflow of each section were routed evenly to subsequent sections, the system states range from 6.2 aircraft to 13.7 aircraft. In particular, the state of section 14 ranges from 11.7 aircraft to 12 aircraft.

Given these initial conditions and capacity constraints, Algorithm 1 was used to generate time-varying routing parameters to solve Problem 1. For this example, $\gamma=0.7$ and $\varepsilon=0.5$. With these values, the stopping criterion was met after 16 iterations. At the final iteration, the cost associated with the Routing LP Problem (24) was zero aircraft $\times$ hour, indicating that the given capacity constraint is not violated under the resulting routing solution.

The given capacity constraint, and resulting capacity bounds and simulated state for section 14 are shown in Figure 4 . The capacity constraint, $\overline{\mathrm{c}}_{14}(t)$, capacity bound $\mathrm{c}_{14}(t)=\sum_{r=1}^{R} c_{14}^{r}(t)$ and full state, $\mathrm{x}_{14}(t)$, of section 14 are shown in Figure 4(a). Capacity bounds $c_{14}^{r}(t)$ and state $x_{14}^{r}(t)$ are also given for $r=1,2,3$ in Figures 4(b), $4(\mathrm{c})$, and $4(\mathrm{~d})$, respectively.

Estimating the network state by piecewise linear $\mathbf{c}(t)$ and outflow rate by a restriction on the linear outflow rate introduces some conservatism into the solution. The difference between the capacity bound and actual state of the system grows throughout the time horizon. At the final time, the state of each section is at most 3.1 aircraft below the bound. Over the whole network, this gap represents $14.3 \%$ of the network capacity. Decreasing the size of the time step $\Delta t$ would reduce this conservatism.

In a second example problem, capacity constraints for section 14 were lowered in order to illustrate the capability of adjusting the capacity constraints if the given capacity constraints cannot be satisfied. All other constraints and parameters are identical to the above example. The given capacity constraint, and resulting capacity bounds and simulated state for section 14 are shown in Figure 5.

Given these initial conditions and capacity constraints, Algorithm 1 was used to generate time-varying routing parameters to solve Problem 1. With these values, the

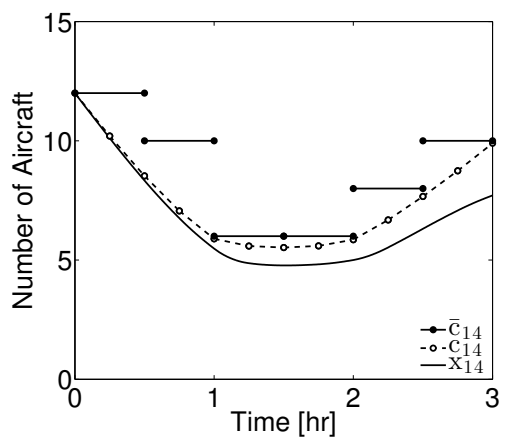

(a) Full state results for section 14 .

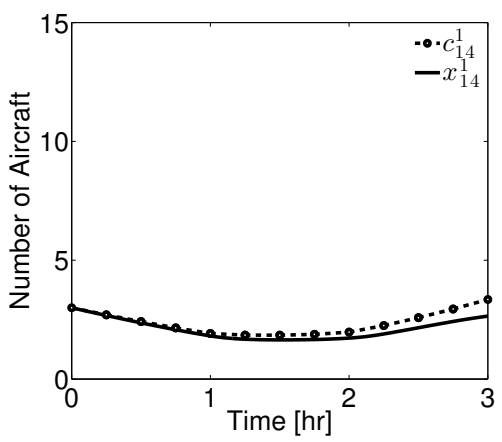

(b) Section 14 destination 1.

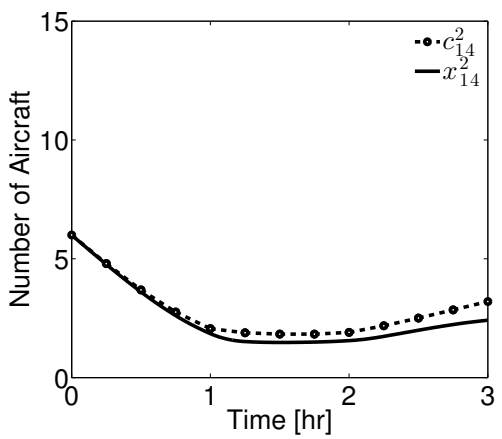

(c) Section 14 destination 2 .

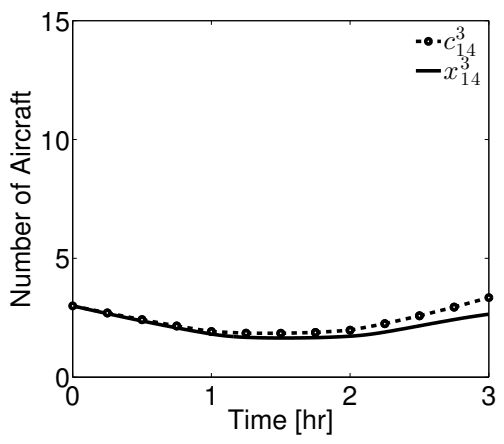

(d) Section 14 destination 3.

Fig. 4. Figure 4(a) shows the capacity constraint $\overline{\mathrm{c}}_{14}(t)$, capacity bound $\mathrm{c}_{14}(t)$ and state $\mathrm{x}_{14}(t)$ of section for 14 for the full network. Figures 4(b), 4(c), and 4(d) show the capacity bound $c_{14}^{r}(t)$ and state $x_{14}^{r}(t)$ of section for 14 for the sub-networks associated with destinations $r=1,2,3$, respectively. 


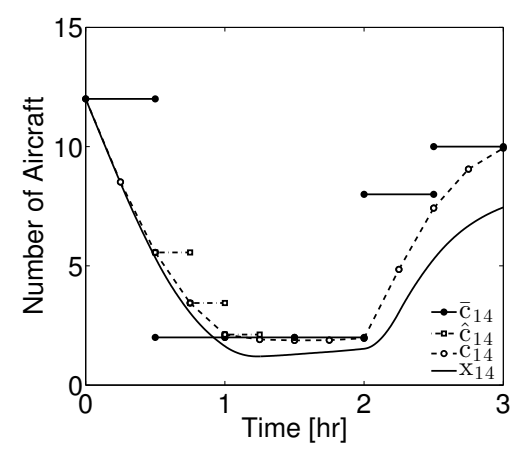

Fig. 5. Capacity constraint $\overline{\mathrm{c}}_{14}$, adjusted capacity constraint $\hat{\mathrm{c}}_{14}$, capacity bound $\mathrm{c}_{14}$ and state $\mathrm{x}_{14}$ of section 14 . Note that $\hat{\mathrm{c}}_{14}$ is only plotted over intervals in which it differs from $\overline{\mathrm{c}}_{14}$.

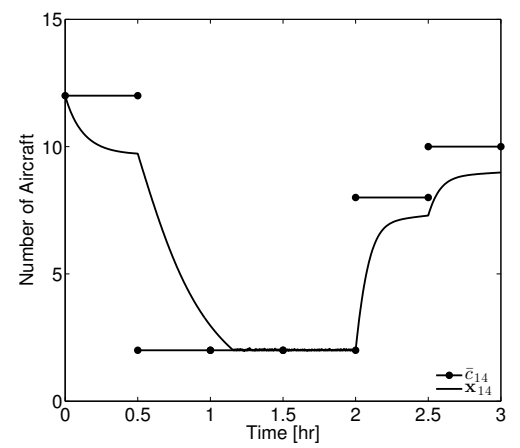

Fig. 6. Capacity constraint $\overline{\mathrm{c}}_{14}$, and state $\mathrm{x}_{14}$ of section 14 resulting from a simple control design technique.

stopping criterion was met after 15 iterations. At the final iteration, the integral of the difference between adjusted constraint $\hat{\mathbf{c}}(t)$ and given constraint $\overline{\mathbf{c}}(t)$, calculated as the cost of the Routing LP Problem (24), is 1.28 aircraft $\times$ hour. Physically, this means that using this solution, the actual section count may exceed the constraint $\overline{\mathbf{c}}(t)$ by no more than an average of 1.28 aircraft over a one hour time period. The state of section 14 falls below the capacity bound at 0.92 hours and violates the capacity constraint by a total of 0.56 aircraft $\times$ hours.

A simple approach to solving this problem would be to route aircraft to subsequent sections proportional to the relative available capacity of the subsequent sections. The resulting state of section 14 is plotted in Figure 6 . This method reacts to changes in the capacity constraint in real time. Thus, when the capacity of section 14 decreases significantly at 30 minutes into the simulation routing parameters are adjusted at this time to reduce the state of section 14. As a result, the state of section 14 falls below the capacity constraint at 1.16 hours and violates the constraint by 2.02 aircraft $\times$ hours.

For each example problem discussed above, the iterative algorithm used to solve for routing parameters terminated in 15 and 14 iterations, respectively. Routing solutions were found for other similar networks with sizes varying from 9 section to 24 sections. The iterative algo- rithm terminated in 10 to 15 iterations for each of these problems.

\section{Conclusions and Future Work}

We addressed the problem of routing design for positive compartmental systems with concave section outflow rates to satisfy time-varying state constraints. The control design technique was presented as an algorithm which can be used to iteratively solve instances of an LP problem, successively tightening bounds on the closedloop system state thus improving the quality of the solution. The resulting routing strategy is a time-varying state-feedback control strategy which guarantees that the state of the closed-loop system remains below the given capacity constraint, or an adjusted capacity constraint if a solution to the given problem cannot be found.

Future work in this area involves the incorporation of uncertain capacity constraints. Such constraints arise in air traffic management problems in which section capacity constraints may depend on weather conditions, which are not deterministic. A control technique which is robust to such uncertainty or reactive in real time to changing constraints would be advantageous for application in this field.

\section{References}

[1] H. Arneson and C. Langbort. A linear programming approach to routing control in networks of constrained linear positive systems. Automatica, 48(5):800 - 807, May 2012.

[2] M. Cantoni, E. Weyer, Y. Li, S.K. Ooi, I. Mareels, and M. Ryan. Control of large-scale irrigation networks. Proceedings of the IEEE, 95(1):75-91, January 2007.

[3] C.F. Daganzo. The cell transmission model: A dynamic representation of highway traffic consistent with the hydrodynamic theory. Transportation Research Part B: Methodological, 28(4):269 - 287, 1994.

[4] N. Dousse, H. Arneson, and C. Langbort. Linear programming based routing design for a positive compartmental system with nonlinear flow rates and piecewise constant capacity constraints. In American Control Conference, pages 4004 - 4009, Montréal, Canada, June 2012.

[5] Y. Fu, H. Wang, C. Lu, and R.S. Chandra. Distributed utilization control for real-time clusters with load balancing. In 27th IEEE International Real-Time Systems Symposium (RTSS'06), pages 137-146, 2006.

[6] J. Krozel, R. Jakobovits, and S. Penny. An algorithmic approach for airspace flow programs. Air Traffic Control Quarterly, 14(3):203-229, 2006. 
[7] J. Le Ny and H. Balakrishnan. Distributed feedback control for an Eulerian model of the National Airspace System. In Proceedings of the 2009 American Control Conference, pages 2891-2897, 2009.

[8] J. Le Ny and H. Balakrishnan. Feedback control of the national airspace system. AIAA Journal of Guidance, Control and Dynamics, 34(3):832, 846 2011.

[9] P. Mazaré, A.H. Dehwah, C.G. Claudel, and A.M. Bayen. Analytical and grid-free solutions to the lighthill-whitham-richards traffic flow model. Transportation Research Part B, 45(10):1727 1748, 2011.

[10] P.K. Menon, G.D. Sweriduk, and K.D. Bilimoria. New approach for modeling, analysis, and control of air traffic flow. Journal of Guidance, Control, and Dynamics, 27(5):737 - 744, September - October 2004.

[11] P.K. Menon, G.D. Sweriduk, T. Lam, G.M. Diaz, and K.D. Bilimoria. Computer-aided Eulerian air traffic flow modeling and predictive control. In AIAA Guidance, Navigation, and Control Conference and Exhibit, August 2004.

[12] H.J. Price (contact). FAA forecast sees continued, steady growth in air travel, March 2015, https://www.faa.gov/news/press_releases/ news_story. cfm?newsId $=18434$

[13] L. Song, C. Wanke, and D. Greenbaum. Predicting sector capacity for TFM decision support. In 6 th AIAA Aviation Technology, Integration and Operations Conference (ATIO), September 2006.

[14] D. Sun, I.S. Strub, and A.M. Bayen. Comparison of the performance of four Eulerian network flow models for strategic air traffic management. Networks and Heterogeneous Media, 2(4):569 - 595, December 2007.

[15] D. Sun, S.D. Yang, I. Strub, A.M. Bayen, B. Sridhar, and K. Sheth. Eulerian trilogy. In Proceedings of the AIAA Guidance, Navigation, and Control Conference and Exhibit. AIAA, August 2006. 